\title{
Estimation and Reduction of Noise from Remotely Sensed Imagery Using Minimum Noise Fraction Techniques
}

\section{Hussein Sabah Jaber*}

Associate Professor, Department of Surveying Engineering, University of Baghdad, Iraq

*Corresponding Author: Hussein Sabah Jaber, Associate Professor, Department of Surveying Engineering, University of Baghdad, Iraq.

Received: March 25, 2019; Published: June 12, 2019

DOI: $10.31080 /$ ASAG.2019.03.0525

\begin{abstract}
Most remotely sensed imagery contains noise caused by atmospheric particles that can obscure the image and make quantitative analysis unreliable. Noise is harmful for data extraction techniques from images. Therefore, this research aims to use Minimum Noise Fraction (MNF) transform technique in ENVI software in order to estimate and Reduce of noise components from images. MNF is an algorithm consisting of two consecutive data reduction operations. The results showed that the first bands (from 1 to 11) have more information with high eigenvalues so there is less noise in these bands after using MNF. Whereas, the last bands (from 20 to 32 ) shows less information due to noise because of low eigenvalue with less information after using MNF. Also, the amount of S/N decrease after applying the inverse MNF rotation because of spectral reduction. It concluded that should select the bands with high Eigenvalues for the MNF inverse transform because these bands have less noise and more information.
\end{abstract}

Keywords: Noise; MNF; S/N; Remotely sensed Imagery; ENVI Software

\section{Introduction}

Noise in images is detrimental for information extraction methods from images such as image classification; absorption band identification, spectral un-mixing, and vegetation cover estimates etc. Noise in images can have many sources and types. Noise may be random distributed over an image, noise can be present in just a few spectral bands or in various bands. The bands in a hyperspectral dataset have differing noise levels $(\mathrm{S} / \mathrm{N})$. It may be desirable to filter or remove those bands that contribute most to noise. When the bands of a hyperspectral dataset have differing amounts of noise, a standard principal components (PC) transform will not produce components with a steadily increasing noise level. This makes it difficult to select a cutoff point [1]. To achieve a components dataset that does have increasing noise (decreasing $\mathrm{S} / \mathrm{N}$ ), a modified PC transform, and termed the Minimum Noise Fraction (MNF) has been developed. The MNF transform is a noise-adjusted principal component transform that estimates and equalizes the amount of noise in each image band to ensure that output components are ordered by their amount of image content. The Minimum Noise Fraction (MNF) transform is an algorithm consisting of two consecutive data reduction operations. The first is based on an estimation of noise in the data as represented by a correlation matrix. This transformation de-correlates and rescales the noise in the data, by variance. At this stage, the information about between band noises has not been considered. The second operation accounts for the original correlations, and creates a set of components that contain weighted information about the variance across all bands in the raw data set [2]. The algorithm retains specific channel information because all original bands contribute to each of the components weighting. Often, most of the surface reflectance variation in a data set can be explained in the first few components, with the rest of the components containing variance as contributed primarily by noise [3].

The objective of this research is presenting a method to reduce the presence of noise in an image by using the MNF transform technique to reduce noise components through ENVI software and evaluate the amount of S/N.

\section{Data used and materials}

A dataset used in this research was A DAIS7915 image collected over an area in southern France. Also, these data was processed using ENVI software. 


\section{Methodology}

There are several steps to do methodology of this research as following:

- $\quad$ Reduce the systematic noise (striping): After this corre ction, evaluate the quality of the image (random noise, and possibly remaining striping) by determining the $\mathrm{S} / \mathrm{N}$ and enhance the $\mathrm{S} / \mathrm{N}$ by a second MNF transform by using ENVI software.

- MNF correction process (Forward MNF): Using ENVI soft ware, open the MNF menu (ENVI: Transform - MNF Rota tion - Forward MNF Rotation -Estimate Noise Statistics from Data) as shown in the figure 1.

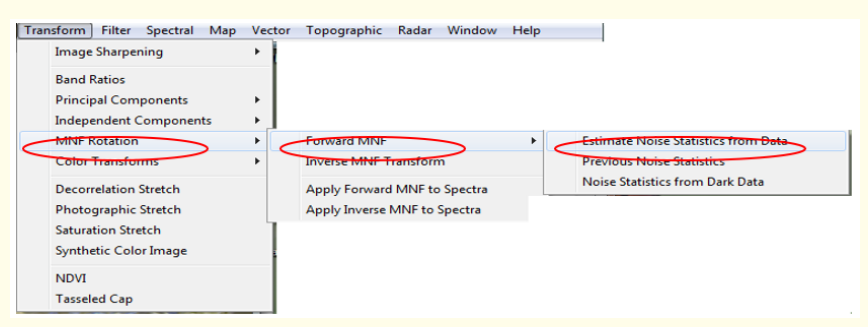

Figure 1: MNF correction process (Forward MNF) using ENVI.

Select p13_Vailhan.bsq, do not set a spatial subset, but do subset the spectral range to the bands belonging to the SWIR detector according to this following and as shown in figure 2. Spectrometer Characteristics (http://www.op.dlr.de/dais/dais-scr.htm)

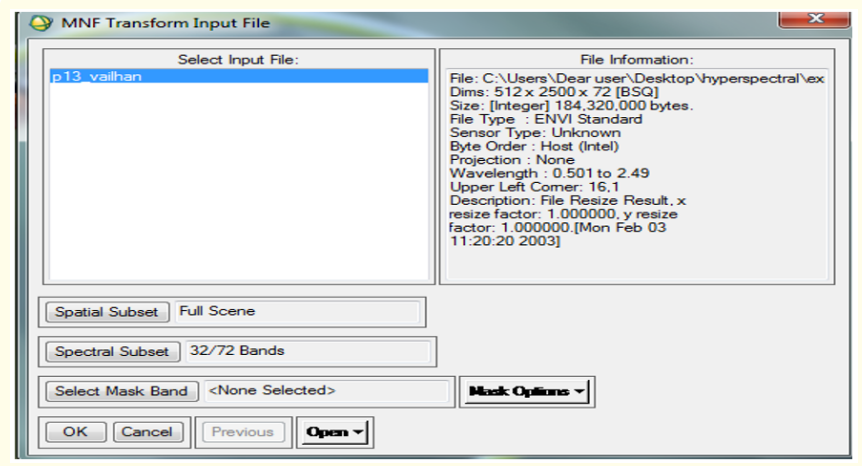

Figure 2: MNF correction process (forward MNF).

1. $400-1000 \mathrm{~nm}: 32$ Bands, Bandwidth $=15-30 \mathrm{~nm}$ Detector: $\mathrm{Si}$

2. $1500-1800 \mathrm{~nm}: 8$ Bands, Bandwidth $=45 \mathrm{~nm}$ Detector: $\mathrm{InSb}$

3. $2000-2500 \mathrm{~nm}: 32$ Bands, Bandwidth $=20 \mathrm{~nm}$ Detector: $\mathrm{InSb}$

4. $3000-5000 \mathrm{~nm}: 1$ Band, Bandwidth $=2.0 \mathrm{Em}$ Detector: $\mathrm{InSb}$

5. $8000-12600 \mathrm{~nm}: 6$ Bands, Bandwidth $=0.9 \mathrm{Em}$ Detector: MCT.
MNF correction process (Inverse MNF)

Using ENVI software, Open again the MNF menu (ENVI: Transform - MNF Rotation - Inverse MNF Rotation) as shown in figure 3.

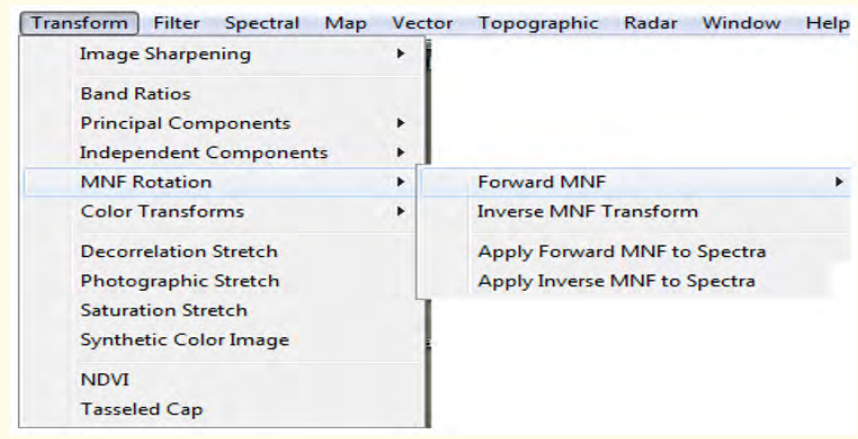

Figure 3: MNF correction process (inverse MNF) using ENVI.

Select the forward-rotated image, subset the band(s) suitable for inverse rotation, select the MNF statistics file that has been calculated in the forward rotation, and output the file to p13_invmnf1. bsq. Repeat this step, but now select only the bands that were disregarded in the previous step as shown in the figure 4 .

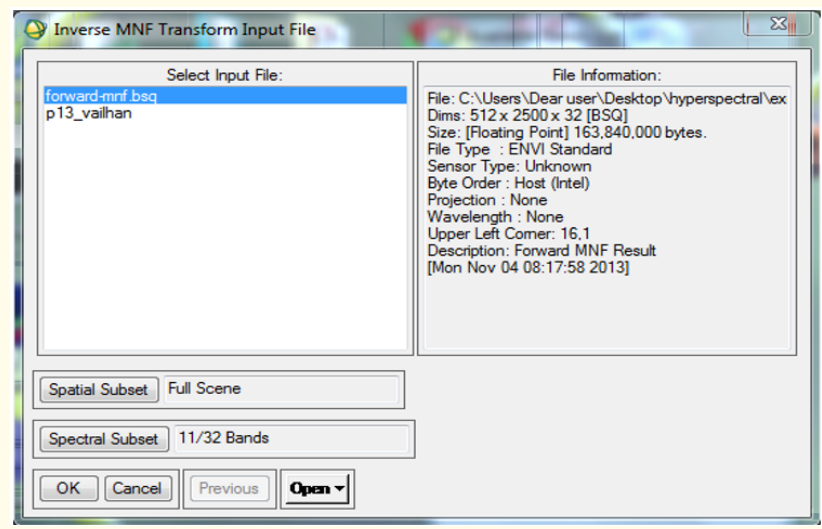

Figure 4: Inverse MNF input file.

Homogeneous area method

Using the homogeneous area method to make an estimate of the $\mathrm{S} / \mathrm{N}$ for a dark and a bright surface in p13_Vailhan.bsq image.

\section{Results and Discussion}

Using MNF technique in ENVI software, a plot showing the Eigenvalues of the DAIS7915 image of the study area as shown in the figure 5 . 


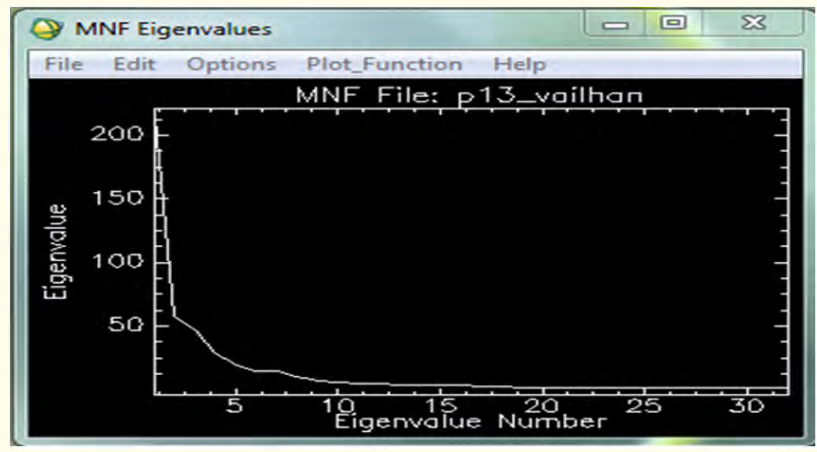

Figure 5: Eigenvalues plot of the DAIS7915 image.

Graph clearly shows the eigenvalue over eigenvalue number. Eigenvalues are Transformation coefficients in principle components analysis that can be used to determine the percent of total variance explained by each of the principle components. In general, the eigenvalues has a downward trend. High eigenvalue shows high information so the 11 first bands have more information compare to others.

The results of MNF correction process (forward MNF) using ENVI software as shown in the figure 6 where it represented as output file of p13_Vailhan.bsq image.

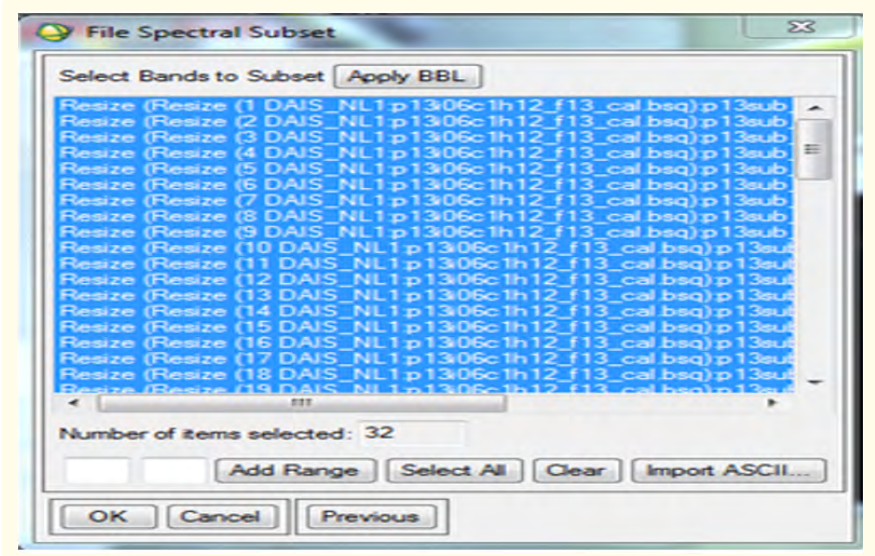

Figure 6: Output file of p13_Vailhan.bsq image.

Also, the results of MNF correction process (inverse MNF) using ENVI software as shown in the figure 7 where it represented as output file of p13_invmnf2.bsq.image.

The results of the bands that contain (mainly) noise and should be disregarded in the inverse MNF transform for P13_invmnf2.bsq image showed the first bands (from 1 to 11 ) have more information with high eigenvalues so there is less noise in these bands. Whereas, the last bands (20 to 32) shows less information due to noise because of low eigenvalue with less information. Evaluate both inverse rotated images and compare them to the original image bands especially 'band animation' will be useful.

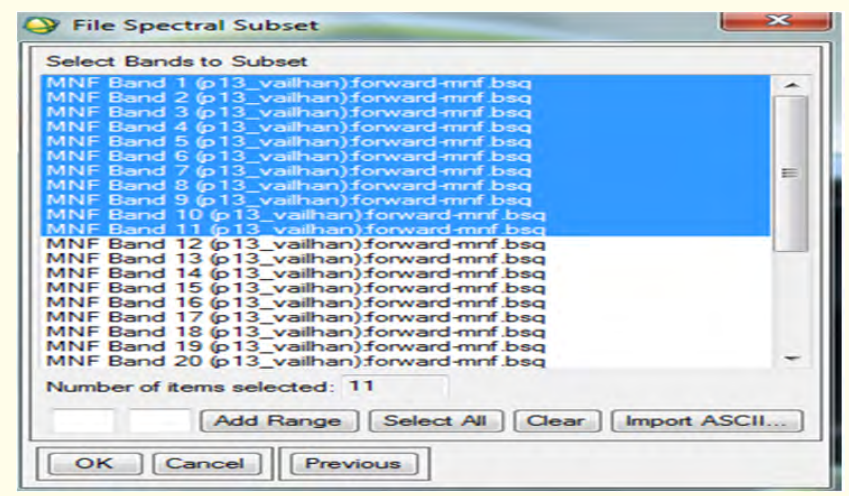

Figure 7: Output file of p13_invmnf2.bsq.image.

From the Eigenvalues plot above, it should select the bands with high Eigenvalues for the MNF inverse transform because these bands have less noise and show more information. It means that the first bands are the bands with more information and less noise as shown in the figure 8.

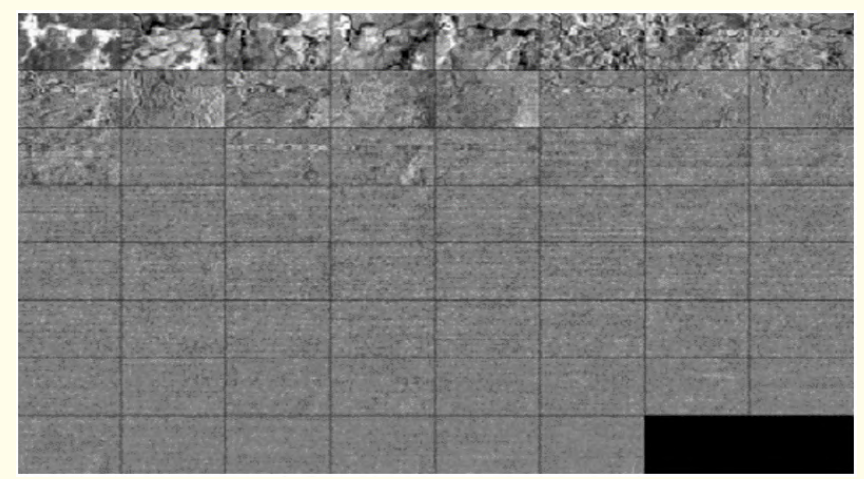

Figure 8: First bands with more information with less noise of the image.

Also, using the homogeneous area method to make an estimate of the $\mathrm{S} / \mathrm{N}$ for a dark and a bright surface in p13_Vailhan.bsq image. The artificial lake can be used as a dark surface and the triangular playground/parking just east of the dam can be used as a bright surface as shown in the figure 9. 


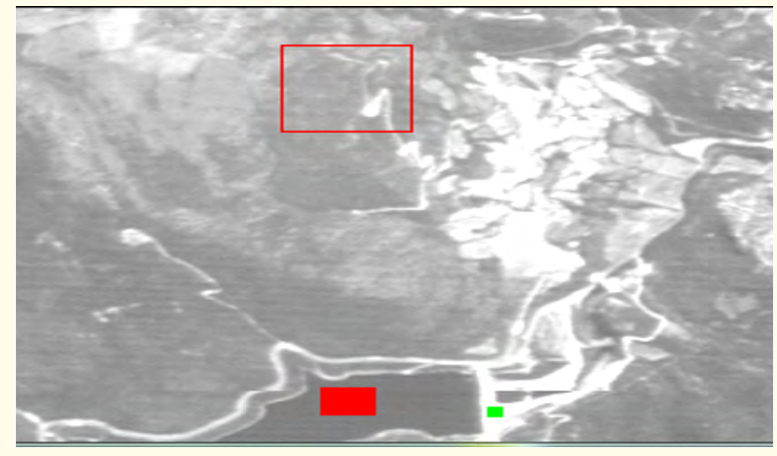

Figure 9: Regions of interest (ROI) image of the study area.

Statistics result of the For ROI (ROI TOOL: Options - Stats All Regions) using ENVI software as shown in the figures (10a, 10b).

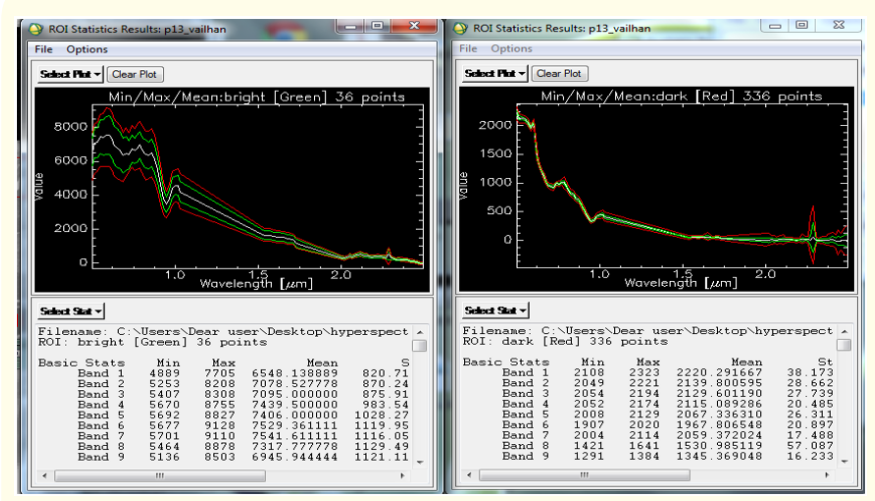

Figure 10: Statistics result of the ROI (dark and bright area).

Figure 10a. Statistics of bright area (green).

Figure 10b. Statistics of dark (red).

The calculations of the $\mathrm{S} / \mathrm{N}$ based on the mean value and standard deviation, (e.g. for bands 5 and 26) where: S/N= Mean/ Standard deviation as shown in the table 1.

\begin{tabular}{|l|c|c|}
\hline \multirow{2}{*}{ Band5 } & Bright area & 7.20 \\
\cline { 2 - 3 } & Dark area & 78.571 \\
\hline \multirow{2}{*}{ Band26 } & Bright area & 7.02 \\
\cline { 2 - 3 } & Dark area & 20.74 \\
\hline
\end{tabular}

Table 1: Calculations of the S/N for bands 5 and 26 using homogeneous area method.
From table 1, it is clear that the bright area has less signal to noise ratio compare to dark area. It means information in bright area is less than information in dark area.

The $\mathrm{S} / \mathrm{N}$ can be enhanced by reducing the random noise in the image. It can focus on the VIS and NIR wavelengths (detector 1 and 2 ), as the quality of the SWIR bands is too low.

Also, it can repeat the MNF transformation for the VIS and NIR bands of the DAIS image and inspect the bands in the MNF domain.

In addition, using the inverse MNF rotation to repeat the calculations of $\mathrm{S} / \mathrm{N}$ for the lake as shown in the table 2.

\begin{tabular}{|l|c|c|}
\hline \multirow{2}{*}{ Band5 } & Bright area & 5.31 \\
\cline { 2 - 3 } & Dark area & 0.477 \\
\hline \multirow{2}{*}{ Band26 } & Bright area & 3.29 \\
\cline { 2 - 3 } & Dark area & 0.245 \\
\hline
\end{tabular}

Table 2: Calculations of the $\mathrm{S} / \mathrm{N}$ for bands 5 and 26 using inverse MNF.

Table 1 and table 2 show S/N in original image and image with applying MNF rotation respectively. The amount of $\mathrm{S} / \mathrm{N}$ decrease because of spectral reduction in MNF so there is less information in compare to original image. Also, the amount of $\mathrm{S} / \mathrm{N}$ decrease after applying the inverse MNF rotation because of spectral reduction [4-12].

\section{Conclusion}

Estimate noise with signal noise ratio and use MNF (Minimum Noise Fraction) to reduce the noise. The advantage of MNF is to order the bands from more information to noisy bands. It concluded that after using MNF showed the first bands have more information with low noise and the last bands showed less information with more noise. The amount of $\mathrm{S} / \mathrm{N}$ decrease because of spectral reduction in MNF so there is less information in compare to original image. Bright area has less signal to noise ratio compare to dark area. It means information in bright area is less than information in dark area. In addition, it concluded that should select the bands with high Eigenvalues for the MNF inverse transform technique because these bands have less noise and more information. 


\section{Bibliography}

1. Michael A and Bhaskar R. ASTER Users Handbook. Version 2.

(2015).

2. Hardin P and Hardin A. "Hyperspectral Remote Sensing of Urban Areas”. Geography Compass 7 (2013): 7-21.

3. Kennedy R., et al. "Remote sensing change detection tools for natural resource managers: Understanding concepts and tradeoffs in the design of landscape monitoring projects". Remote sensing of environment 113 (2009): 1382-1396.

4. Delwiche SR. "Classification of scab and other mold-damaged wheat kernels by near infrared reflectance spectroscopy". Transactions of the ASAE 46 (2003): 731-738.

5. Envi User's Guide.

6. Graham RL. "An efficient algorithm for determining the convex hull of a finite planar set". Information Processing Letters 1 (1972): 132-133.

7. Lillesand TM and Kiefer RW. Remote Sensing and Image Interpretation, 3rd Ed., John Wiley and Sons, Inc.: Toronto (1994).

8. Lattin JM., et al. "Analyzing multivariate data". china: Machine Press (2003): 38-40.

9. Lu D., et al. "Change detection techniques". International journal of remote sensing 25 (2004): 2365-2401.

10. Naidu RA., et al. "The potential of spectral Reflectance technique for the detection of Grapevine leafroll-associated virus-3 in Two red-berried wine grape cultivars". Computers and Electronics in Agriculture 66 (2009): 38-45.

11. Nutter FW., et al. "Assessing the accuracy, intrarater repeatability, and inter-rater reliability of disease assessment systems". Phytopathology 83 (1993): 806-812.

12. USGS and Japan ASTER Program. ASTER scene AST L1B_003_06262000100635, 1B, USGS, Sioux Falls, 6/26/2001 (2003).

Volume 3 Issue 7 July 2019

(C) All rights are reserved by Hussein Sabah Jaber. 